\title{
Four-loop singularities of the massless fermion propagator in quenched three-dimensional QED
}

\author{
A. F. Pikelner $\odot^{1, *}$ V.P. Gusynin $\oplus^{2, \dagger}$ A. V. Kotikov $\oplus^{1, \$}$ and S. Teber $\odot^{3, \S}$ \\ ${ }^{1}$ Bogoliubov Laboratory of Theoretical Physics, Joint Institute for Nuclear Research, \\ 141980 Dubna, Russia \\ ${ }^{2}$ Bogolyubov Institute for Theoretical Physics, Kyiv 03143, Ukraine \\ ${ }^{3}$ Sorbonne Université, CNRS, Laboratoire de Physique Théorique et Hautes Energies, \\ LPTHE, F-75005 Paris, France
}

(Received 4 September 2020; accepted 8 October 2020; published 10 November 2020)

\begin{abstract}
We calculate the three- and four-loop corrections to the massless fermion propagator in threedimensional quenched quantum electrodynamics with four-component fermions. The three-loop correction is finite and gauge invariant but the four-loop one has singularities except in the Feynman gauge where it is also finite. Our results explicitly show that, up to four loops, gauge-dependent terms are completely determined by lower order ones in agreement with the Landau-Khalatnikov-Fradkin transformation.
\end{abstract}

DOI: $10.1103 /$ PhysRevD.102.105012

\section{INTRODUCTION}

Three-dimensional quantum electrodynamics $\left(\mathrm{QED}_{3}\right)$ is an archetypal gauge field theory model of strongly interacting relativistic planar fermions. In Euclidean space, it is described by the action

$$
S=\int \mathrm{d}^{3} x\left[\frac{1}{4} F_{\mu \nu}^{2}+\bar{\Psi}_{i \gamma_{\mu}} D_{\mu} \Psi_{i}\right]
$$

where $D_{\mu}=\partial_{\mu}-i e A_{\mu}, i=1,2, \ldots n_{f}$ with $n_{f}$ the number of four-component massless Dirac fermion flavors, Euclidean gamma matrices satisfy $\gamma_{\mu}^{\dagger}=\gamma_{\mu},\left\{\gamma_{\mu}, \gamma_{\nu}\right\}=\delta_{\mu \nu}$ and the gauge coupling constant $e^{2}$ has dimension of mass.

For the past 40 years, this super-renormalizable model served as a toy model for exploring several key problems in quantum field theory such as infrared (IR) singularities in low-dimensional massless particle theories [1-5] (see recent progress in $[6,7]$ ) and dynamical symmetry breaking and fermion mass generation [8-18] (see recent progress in [19-23]). In the last 30 years, considerable interest in $\mathrm{QED}_{3}$ also came from its applications to condensed matter physics systems with relativisticlike gapless quasiparticle

\footnotetext{
*pikelner@theor.jinr.ru

vgusynin@bitp.kiev.ua

kotikov@theor.jinr.ru

${ }_{\text {teber@lpthe.jussieu.fr }}$
}

Published by the American Physical Society under the terms of the Creative Commons Attribution 4.0 International license. Further distribution of this work must maintain attribution to the author(s) and the published article's title, journal citation, and DOI. Funded by SCOAP ${ }^{3}$. excitations at low-energies such as high- $T_{c}$ superconductors [24-26], planar antiferromagnets [27], and graphene [28] (for graphene, see reviews in Ref. [29]).

A slight simplification of $\mathrm{QED}_{3}$ takes place in the so-called quenched limit in which closed fermion loops are set to zero (this amounts to take $n_{f}=0$ ). This limit arose as a useful approximation in the study of the lattice representation of four-dimensional quantum chromodynamics $\left(\mathrm{QCD}_{4}\right)$, see [30], where it was shown that a reasonable estimate of the hadron spectrum could be obtained by eliminating all internal quark loops. The quenched limit, and other approximation schemes such as the ladder (rainbow) approximation, were used in $\mathrm{QED}_{4}$ for a long time to try solving nonperturbatively a more manageable truncated set of Schwinger-Dyson equations (see Refs. [31-33] and references therein to earlier papers). The quenched approximation in $\mathrm{QED}_{4}$ is now still in use in order to include QED effects in lattice QCD calculations (see the recent Ref. [34] and discussions therein).

In a recent paper [7], we studied the gauge covariance of the massless fermion propagator of quenched $\mathrm{QED}_{3}$ in a linear covariant gauge in dimensional regularization (following Refs. [33,35,36]). At this point, let us note that, as in the four-dimensional case, both the fermion propagator and vertex function of $\mathrm{QED}_{3}$ possess the important property of being covariant under the Landau-KhalatnikovFradkin (LKF) transformations $[37,38]$. These transformations, which have a simple form in coordinate space, allow one to compute Green's functions in an arbitrary covariant gauge provided they are known in a particular gauge (for applications of the LKF transformations to $\mathrm{QED}_{3}$, see the papers [39] and the review in Ref. [40]). 
The analysis of the LKF transformation of the massless fermion propagator of quenched $\mathrm{QED}_{3}$ carried out in [7], led us to reconnect with the long-standing issue of IR singularities in $\mathrm{QED}_{3}$. In particular, we concluded that, exactly in three dimensions $(d=3)$, all of the odd perturbative coefficients of the massless fermion propagator, starting from the third order one, have to vanish in any gauge in order for quenched $\mathrm{QED}_{3}$ to be free of (IR) singularities. It turns out that there is a widespread opinion that quenched perturbation theory is IR finite [1] and recent lattice studies of quenched $\mathrm{QED}_{3}$ seem to confirm it [6]. Usually, the presence of IR divergences is related to fermion loops. This is because, for dimensional reasons, higher-order diagrams contain higher powers of momentum in the denominator. For example, the two-loop fermion selfenergy diagram with vacuum polarization gives rise to a logarithmic divergence [1] which corresponds to a $1 / \varepsilon$ pole in dimensional regularization in $d=3-2 \varepsilon$ [2]. However, for the same dimensional reasons, higherorder quenched diagrams can lead to IR divergences, too. Indeed, it is easy to see that, at four loops, e.g., the diagram with an insertion of three one-loop fermion self-energies into a fermion line is logarithmically divergent in gauges different from the Landau gauge (this was for the first time mentioned in Ref. [3]). The question then is whether IR divergences of separate diagrams cancel in their sum or not. The LKF transformation by itself is unable to provide explicit values for the coefficients in a given gauge. Order by order calculations are therefore required (at least in a given gauge) in order to analyze the IR finiteness of $\mathrm{QED}_{3}$ in accordance with our statement above.

In the present paper, we undertake this task and explicitly calculate the fermion propagator of quenched $\mathrm{QED}_{3}$ at three and four loops in an arbitrary linear covariant gauge and in dimensional regularization in $d=3-2 \varepsilon$. We find that the three-loop correction is finite and gauge invariant. Accordingly, the four-loop one has singularities. Our exact results show that, up to four loops, gauge-dependent terms are completely determined by lower order ones in perfect agreement with properties of the LKF transformation following the study [7].

The paper is organized as follows. In Sec. II, we specify our notations, provide some details of the calculations and present the results for the three and four-loop corrections to the fermion propagator. In Sec. III, we briefly recall the LKF transformation for the fermion propagator in momentum space and check its consistency with our four-loop perturbative results. Some predictions beyond four-loops are also presented. In Sec. IV, a representative sample of the computed diagrams is presented by focusing on the Landau gauge. The results are summarized and discussed in Sec. V.

\section{FERMION PROPAGATOR: THREE- AND FOUR-LOOP COEFFICIENTS}

\section{A. Notations}

In the following, we shall consider a Euclidean space of dimension $d=3-2 \varepsilon$. The general form of the fermion propagator $S_{F}(p, \xi)$ in some gauge $\xi$ reads:

$$
S_{F}(p, \xi)=\frac{\mathrm{i}}{\not p} P(p, \xi),
$$

where the tensorial structure, e.g., the factor $\not p$ containing Dirac $\gamma$ matrices, has been extracted and $P(p, \xi)$ is a scalar function of $p=\sqrt{p^{2}}$.

It is convenient to first express $P(p, \xi)$ as

$$
P(p, \xi)=\frac{1}{1-\sigma(p, \xi)},
$$

where the one-particle-irreducible part, $\sigma(p, \xi)$, can be represented as

$$
\sigma(p, \xi)=\sum_{m=1}^{\infty} \sigma_{m}(\xi)\left(\frac{\alpha}{2 \sqrt{\pi} p}\right)^{m}\left(\frac{\bar{\mu}^{2}}{p^{2}}\right)^{m \varepsilon} .
$$

Here, $\sigma_{m}(\xi)$ are the coefficients of the loop expansion of the fermion self-energy, $\alpha=e^{2} /(4 \pi)$ is the dimensionful coupling constant and $\bar{\mu}$ is the $\overline{\mathrm{MS}}$ scale.

Following our previous paper [7], the fermion propagator can be equivalently represented as

$$
P(p, \xi)=\sum_{m=0}^{\infty} a_{m}(\xi)\left(\frac{\alpha}{2 \sqrt{\pi} p}\right)^{m}\left(\frac{\bar{\mu}^{2}}{p^{2}}\right)^{m \varepsilon}
$$

where $a_{m}(\xi)$ are now the coefficients of the loop expansion of $P(p, \xi)$. As will be seen in Sec. III, the form (5) is convenient to study the properties of the propagator under the LKF transformation.

In both Eqs. (4) and (5), the expansion has been written in terms of the dimensionless ratio $\alpha / p$ with an additional conventional factor of $1 /(2 \sqrt{\pi})$. Its exact form is coming from the consideration of four-dimensional QED in [35] (see also Ref. [7] and discussions therein). Up to four loops, the coefficients $a_{m}(\xi)$ and $\sigma_{m}(\xi)$ are related to each other as

$$
\begin{aligned}
& a_{1}=\sigma_{1}, \quad a_{2}=\sigma_{2}+\sigma_{1}^{2}, \quad a_{3}=\sigma_{3}+2 \sigma_{2} \sigma_{1}+\sigma_{1}^{3}, \\
& a_{4}=\sigma_{4}+2 \sigma_{3} \sigma_{1}+\sigma_{2}^{2}+3 \sigma_{2} \sigma_{1}^{2}+\sigma_{1}^{4} .
\end{aligned}
$$

\section{B. Calculational details}

In quenched QED at one, two, three, and four loops we encountered 1,2,10, and 74 fermion self-energy diagrams, respectively. Let us note that the two-loop diagrams of 
$\mathrm{QED}_{3}$ were considered earlier in $[1,2]$. These papers mainly focused on the IR divergent two-loop diagram (with a fermion loop insertion) which is absent in the quenched case. In [2], higher order diagrams were considered but still with fermion loop insertions. To the best of our knowledge, the two-loop quenched $\mathrm{QED}_{3}$ fermion propagator was calculated in [39]. Moreover, all two-loop diagrams of $\mathrm{QED}_{3}$ were computed in [41] (see also the last paper in [29]) and their $\varepsilon$ expansion provided near $d=3$. The fact that the first singularities in the fermion propagator of $\mathrm{QED}_{3}$ without vacuum polarization arise at four loops was mentioned for the first time in Ref. [3]. However, three- and four-loop corrections to the quenched $\mathrm{QED}_{3}$ fermion propagator have not been previously computed. As will be shown in the next subsections, the three-loop correction is finite but IR singular diagrams do appear at four loops in the quenched case (in agreement with [3]) and there are 42 of them, the sum of which will be analyzed in the following.

In order to compute all of these diagrams and extract from them the unrenormalized fermion self-energy of $\mathrm{QED}_{3}$ up to four loops, we first considered the corresponding results for the unrenormalized QCD quark propagator. The exact expression for the latter, written in terms of a set of master integrals and valid for arbitrary space-time dimension $d$ and arbitrary gauge-fixing parameter $\xi$, is available up to four loops from [42] and also shipped with the FORCER package [43] designed for the reduction of four-loop massless propagator-type integrals. The fermion propagator of $\mathrm{QED}_{d}$ is obtained from this $\mathrm{QCD}_{d}$ result upon performing the following substitutions:

$$
\begin{aligned}
& C_{A}=d_{A}^{a b c d} d_{A}^{a b c d}=d_{A}^{a b c d} d_{F}^{a b c d}=0, \\
& C_{F}=d_{F}^{a b c d} d_{F}^{a b c d}=T_{F}=1 .
\end{aligned}
$$

After that, the quenched limit of $\mathrm{QED}_{d}$ is obtained by setting $n_{f}=0$ which discards all diagrams with closed fermion loops.

The main remaining task was then to compute all required propagator-type master integrals in an $\varepsilon$ expansion around $d=3(\varepsilon=(3-d) / 2)$. This could be achieved with the help of the dimensional recurrence and analyticity (DRA) method [44], which expresses the integrals in the form of fast convergent sums. The latter are then evaluated with high-precision numerical values. This in turn allows us to reconstruct the analytic expression of master integrals (in any space-time dimension) with the help of the PSLQ algorithm [45] once an adequate basis of transcendental constants is defined.

We note that near $d=4(\varepsilon=(4-d) / 2)$, such calculations yield the expansions of all needed masters [46]. The results are well-known and available in input form for the SUMMERTIME package [47] with the package itself and also from [48].
The case $d=3-2 \varepsilon$ is less well known and was considered in the paper [47] from which the $\varepsilon$ expansion of most of the needed master integrals for the current calculation is available. The successful reconstructions of [47] around $d=3$, were carried out using a basis of transcendental constants consisting only of multiple zeta values (MZV) and alternating MZVs. As remarked already in [47], such a basis is too restrictive to enable the representation of all of the masters and some of them were left unreconstructed.

In our work we successfully reconstructed all the needed integrals and found agreement with results of [46] using a basis consisting of MZV and alternating MZVs. On top of that, we encountered one of the constants left unknown in [47]. By a careful analysis of the representation of one such integrals with known closed form expressions in the form of the ${ }_{3} F_{2}$ functions [41], ${ }^{1}$ we found that elements of its $\varepsilon$ expansion belong to the set of generalized polylogarithms (GPLs) with fourth-root of unity alphabet. Extending our PSLQ basis to include the full set of GPLs with fourth root of unity arguments we successfully reconstructed its analytical value

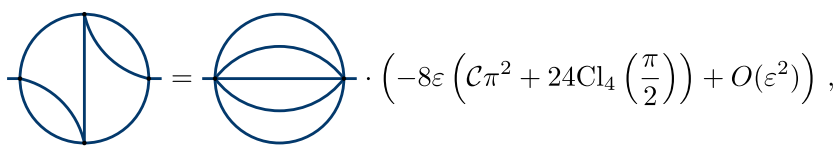

where we factored out the four-loop sunset integral to follow the normalization prescriptions of [47]. In Eq. (8), $\mathcal{C}=\mathrm{Cl}_{2}(\pi / 2)$ is Catalan's constant and $\mathrm{Cl}_{\mathrm{n}}(\theta)$ is Clausen's function which, for even weight, can be expressed through the classical polylogarithm as $\mathrm{Cl}_{2 k}(\theta)=\operatorname{ImLi}_{2 k}\left(e^{i \theta}\right)$. As can be understood from the above result, the required extension of the basis of transcendental constants includes polylogarithms with fourth-root of unity argument, in the present case Clausen's function (see, for example, Ref. [50], where GPLs with second, fourth, and sixth root of unity arguments appear).

\section{Results for the fermion self-energy (4)}

We now present our results for $\sigma_{m}(\xi)$, which can be represented as

$$
\sigma_{m}(\xi)=\sigma_{m}(0)+\xi \tilde{\sigma}_{m}(\xi),
$$

where we have explicitly separated the part independent from $\xi$ which corresponds to the full result in the Landau gauge.

\footnotetext{
${ }^{1}$ The results of [41] were obtained based on the general approach of Ref. [49], where a class of more complicated diagrams with three arbitrary indices was studied and the corresponding results were expressed in terms of combinations of ${ }_{3} F_{2}$-hypergeometric functions of unit argument.
} 
Considering the first two orders of the $\varepsilon$ expansion, we have for the coefficients $\sigma_{m}(0)$

$$
\begin{aligned}
\sigma_{1}(0)= & 0 \\
\sigma_{2}(0)= & \pi\left[\frac{3 \pi^{2}}{4}-7-\left(\left(1-3 l_{2}\right) \pi^{2}+12\right) \varepsilon\right] \\
\sigma_{3}(0)= & \pi^{5 / 2}\left[\frac{43 \pi^{2}}{4}-105+\varepsilon\left\{2\left(185-105 l_{2}+137 \zeta_{3}\right)\right.\right. \\
& \left.\left.-\frac{\pi^{2}}{6}\left(451-171 l_{2}\right)\right\}\right] \\
\sigma_{4}(0)= & \pi^{2}\left[\left(\frac{43}{6} \pi^{2}-70\right) \frac{1}{\varepsilon}+\bar{\sigma}_{4}+\frac{5954}{3}+\frac{173}{18} \pi^{2}\right. \\
& \left.-\frac{513}{10} \pi^{4}\right]
\end{aligned}
$$

where $\bar{\sigma}_{4}$ contains the most complicated part

$$
\begin{aligned}
\bar{\sigma}_{4}= & 209 l_{2}^{4}+5016 a_{4}+4264 \mathrm{Cl}_{4}(\pi / 2) \\
& +\left(\frac{533}{3} \mathcal{C}-930 l_{2}\right) \pi^{2}+\frac{2078}{3} \zeta_{3},
\end{aligned}
$$

and

$$
l_{2}=\ln 2, \quad a_{4}=\mathrm{Li}_{4}(1 / 2), \quad \zeta_{n}=\mathrm{Li}_{n}(1),
$$

where $\mathrm{Li}_{n}$ are polylogarithms.

With the same accuracy, we have for the coefficients $\tilde{\sigma}_{m}(\xi)$

$$
\begin{aligned}
\tilde{\sigma}_{1}(\xi)= & -\frac{\pi^{3 / 2}}{2}\left(1-2\left(1-l_{2}\right) \varepsilon\right) \\
\tilde{\sigma}_{2}(\xi)= & \pi \xi\left[1-\frac{\pi^{2}}{4}-\left(4-\left(1-l_{2}\right) \pi^{2}\right) \varepsilon\right] \\
\tilde{\sigma}_{3}(\xi)= & \pi^{5 / 2}\left[\frac{3 \pi^{2}}{4}-7+\left(1-\frac{\pi^{2}}{8}\right) \xi^{2}\right. \\
& +\varepsilon\left\{-40-14 l_{2}+\frac{\pi^{2}}{2}\left(4+9 l_{2}\right)\right. \\
& \left.\left.+\left(2 l_{2}-4+\frac{3 \pi^{2}}{4}\left(1-l_{2}\right)\right) \xi^{2}\right\}\right] \\
\tilde{\sigma}_{4}(\xi)= & \pi^{2}\left[\left(70-\frac{43 \pi^{2}}{6}\right) \frac{1}{\varepsilon}+\frac{520}{3}-\frac{\pi^{2}}{9}\left(881+42 l_{2}\right)\right. \\
& +\frac{129 \pi^{4}}{27}-\frac{548}{3} \zeta_{3}+\xi\left(28-\frac{33 \pi^{2}}{4}+\frac{9 \pi^{4}}{16}\right) \\
& \left.+\xi^{3}\left(-\frac{4}{3}+\frac{3 \pi^{2}}{4}-\frac{\pi^{4}}{16}\right)\right] .
\end{aligned}
$$

We would like to note that the finite parts $(\epsilon=0)$ of the coefficients $\sigma_{1}(\xi)$ and $\sigma_{2}(\xi)$ coincide with the corresponding ones in Ref. [41].

Moreover, from Eqs. (10d) and (13d), we notice that

$$
\sigma_{4}(\xi)=\pi^{2}\left(\frac{43}{6} \pi^{2}-70\right) \frac{(1-\xi)}{\varepsilon}+O\left(\varepsilon^{0}\right),
$$

i.e., the total four-loop contribution is finite in the Feynman gauge.

\section{Results for the fermion propagator (5)}

As in the case of $\sigma_{m}(\xi)$ in (9), it is convenient to present the results for $a_{m}(\xi)$ in the form

$$
a_{m}(\xi)=a_{m}(0)+\xi \tilde{a}_{m}(\xi)
$$

where we have also explicitly separated the part independent from $\xi$ which corresponds to the full result in the Landau gauge.

Since $\sigma_{1}(\xi) \sim \xi$, we see from (6) that $a_{i}(0)=\sigma_{i}(0)$ for $i \leq 3$ and thus $a_{i}(0)$ with $i \leq 3$ can straightforwardly be read off from Eqs. (10a), (10b), and (10c). In agreement with (6), we have for $a_{4}(0)$

$$
\begin{aligned}
a_{4}(0) & =\sigma_{4}(0)+\pi^{2}\left(\frac{3 \pi^{2}}{4}-7\right)^{2}, \\
& =\pi^{2}\left[\left(\frac{43}{6} \pi^{2}-70\right) \frac{1}{\varepsilon}+\bar{\sigma}_{4}+\frac{6101}{3}-\frac{8}{9} \pi^{2}-\frac{4059}{80} \pi^{4}\right],
\end{aligned}
$$

where $\bar{\sigma}_{4}$ was defined in Eq. (11).

With the same accuracy, we have for the coefficients $\tilde{a}_{m}(\xi)$

$\tilde{a}_{1}(\xi)=\tilde{\sigma}_{1}(\xi)=-\frac{\pi^{3 / 2}}{2}\left(1-2\left(1-l_{2}\right) \varepsilon\right)$,

$\tilde{a}_{2}(\xi)=\pi \xi(1-4 \varepsilon)$,

$\tilde{a}_{3}(\xi)=\pi^{5 / 2} \varepsilon\left(\frac{43 \pi^{2}}{4}-105+2 \xi^{2}\right)$,

$$
\begin{aligned}
\tilde{a}_{4}(\xi)= & \frac{\pi^{2}}{3}\left[\left(210-\frac{43 \pi^{2}}{2}\right) \frac{1}{\varepsilon}+520+\frac{2 \pi^{2}}{3}\left(32-21 l_{2}\right)\right. \\
& \left.-548 \zeta_{3}+6 \xi\left(7-\frac{3 \pi^{2}}{4}\right)-\xi^{3}\right] .
\end{aligned}
$$

We would like to note that the finite parts $(\epsilon=0)$ of the coefficients $a_{1}(\xi)$ and $a_{2}(\xi)$ coincide with the corresponding ones in Ref. [39] (see also Ref. [7] and discussions therein). 
From the above results, we see that the coefficients $\tilde{a}_{m}(\xi)$ $(m=2,3,4)$ have simpler forms than the corresponding coefficients $\tilde{\sigma}_{m}(\xi)$. Moreover, as in the case of $\sigma_{4}(\xi)$, we notice from Eqs. (16) and (17d) that

$$
\begin{aligned}
a_{4}(\xi) & =\sigma_{4}(\xi)+O\left(\varepsilon^{0}\right), \\
& =\frac{2 \pi^{2}}{3}\left(\frac{43 \pi^{2}}{4}-105\right)(1-\xi) \frac{1}{\varepsilon}+O\left(\varepsilon^{0}\right),
\end{aligned}
$$

i.e., the total four-loop contribution is finite in the Feynman gauge.

\section{LKF TRANSFORMATION}

\section{A. Comparison with the perturbative results up to four loops}

It is convenient to introduce the $x$-space representation $S_{F}(x, \xi)$ of the fermion propagator as

$$
S_{F}(x, \xi)=\not \chi X(x, \xi),
$$

which is related by the Fourier transform to $S_{F}(p, \xi)$ in (2). The LKF transformation expresses the covariance of the fermion propagator under a gauge transformation. It can be derived by standard arguments, see, e.g., [37,38], and its general form can be written as (see Refs. [35,36]):

$$
\begin{aligned}
S_{F}(x, \xi) & =S_{F}(x, \eta) e^{D(x)}, \\
D(x) & =e^{2} \Delta \mu^{2 \varepsilon} \int \frac{\mathrm{d}^{d} q}{(2 \pi)^{d}} \frac{e^{-i q x}}{q^{4}}, \quad \Delta=\xi-\eta,
\end{aligned}
$$

in $d=3-2 \varepsilon$. The calculation [33] yields:

$$
D(x)=-\frac{\alpha \Delta}{2 \pi \mu} \frac{\Gamma(1 / 2-\varepsilon)}{1+2 \varepsilon}\left(\pi \mu^{2} x^{2}\right)^{1 / 2+\varepsilon} .
$$

The LKF transformation (20) relates [7] the coefficients $a_{k}(\xi)$ and $a_{m}(\xi)$ in (5) as

$$
a_{k}(\xi)=\sum_{m=0}^{k}(-2 \Delta)^{k-m} a_{m}(\eta) \Phi(m, k, \varepsilon) \phi(k-m, \varepsilon),
$$

where

$$
\Phi(m, k, \varepsilon)=\frac{\Gamma(3 / 2-m / 2-(m+1) \varepsilon) \Gamma(1+k / 2+k \varepsilon)}{\Gamma(1+m / 2+m \varepsilon) \Gamma(3 / 2-k / 2-(k+1) \varepsilon)},
$$

and

$$
\phi(l, \varepsilon)=\frac{\Gamma^{l}(1 / 2-\varepsilon)}{l !(1+2 \varepsilon)^{l} \Gamma^{l}(1+\varepsilon)} .
$$

Consider $a_{m}(\xi)$ with $m \leq 4$. Keeping only the first two orders of the $\varepsilon$ expansion, we have:

$$
\begin{aligned}
a_{0}(\xi)= & a_{0}(\eta)=1 \\
a_{1}(\xi)= & a_{1}(\eta)-\frac{\pi}{2} \delta\left(1+2 \varepsilon\left(l_{2}-1\right)\right) a_{0}(\eta) \\
a_{2}(\xi)= & a_{2}(\eta)-\frac{4}{\pi} \delta\left(1-2 \varepsilon\left(l_{2}+1\right)\right) a_{1}(\eta) \\
& +\delta^{2}(1-4 \varepsilon) a_{0}(\eta) \\
a_{3}(\xi)= & a_{3}(\eta)+6 \pi \varepsilon \delta a_{2}(\eta)-12 \varepsilon \delta^{2} a_{1}(\eta) \\
& +2 \pi \varepsilon \delta^{3} a_{0}(\eta) \\
a_{4}(\xi)= & a_{4}(\eta)-\frac{2 \delta}{3 \pi \varepsilon}\left(1+2 \varepsilon\left(3-l_{2}\right)\right) a_{3}(\eta)-2 \delta^{2} a_{2}(\eta) \\
& +\frac{8 \delta^{3}}{3 \pi} a_{1}(\eta)-\frac{\delta^{4}}{3} a_{0}(\eta)
\end{aligned}
$$

where $\delta=\sqrt{\pi} \Delta$.

Setting $\eta=0$, i.e., choosing the initial gauge as the Landau gauge, we can see that our results for $\tilde{a}_{m}(\xi)$ are completely determined by $a_{l}(\xi),(l<m)$, i.e., by the coefficients of lower orders in agreement with the properties of the LKF transformation.

Moreover, the results of Eqs. (25) are in full agreement with the perturbative results presented in Sec. II D.

\section{B. Beyond four loops}

As can be seen from (18), the singularity of the four-loop coefficient $a_{4}(\xi)$ is $\sim(1-\xi)$, i.e., the fermion propagator including up to four-loop corrections is finite in the Feynman gauge. This intriguing fact calls for a closer examination of higher order contributions and, as a first try, we will proceed by using the LKF transformation.

We therefore consider $a_{5}(\xi)$ and $a_{6}(\xi)$. From Eq. (22), we have:

$$
\begin{aligned}
a_{5}(\xi)= & a_{5}(\eta)+\frac{45}{2} \pi \varepsilon \delta a_{4}(\eta)-\frac{15}{2} \delta^{2} a_{3}(\eta)-15 \pi \varepsilon \delta^{3} a_{2}(\eta) \\
& +15 \varepsilon \delta^{4} a_{1}(\eta)-\frac{3}{2} \pi \varepsilon \delta^{5} a_{0}(\eta), \\
a_{6}(\xi)= & a_{6}(\eta)+\frac{4 \delta}{5 \pi \varepsilon} a_{5}(\eta)-9 \delta^{2} a_{4}(\eta)+\frac{2 \delta^{3}}{\pi \varepsilon} a_{3}(\eta) \\
& +3 \delta^{4} a_{2}(\eta)-\frac{12 \delta^{5}}{5 \pi} a_{1}(\eta)+\frac{\delta^{6}}{5} a_{0}(\eta) .
\end{aligned}
$$

We may then take the $\eta$ gauge as the Feynman gauge and consider $a_{5}(\xi)$ and $a_{6}(\xi)$ with accuracies $O(\varepsilon)$ and $O\left(\varepsilon^{0}\right)$, respectively. This yields

$a_{5}(\xi)=a_{5}(1)-\frac{15}{2} \pi(\xi-1)^{2} a_{3}+O(\varepsilon)$, 


$$
\begin{aligned}
a_{6}(\xi)= & a_{6}(1)+\frac{4(\xi-1)}{5 \sqrt{\pi} \varepsilon} a_{5}(1)+\frac{2 \sqrt{\pi}(\xi-1)^{3}}{\varepsilon} a_{3} \\
& +O\left(\varepsilon^{0}\right),
\end{aligned}
$$

where we took into account the fact that the finite part of $a_{3}$ is gauge-independent.

From these results, we see that the LKF transformation gives information about the $\xi$ dependence of $a_{5}(\xi)$ and $a_{6}(\xi)$, as expected. Some singularities may still be hidden in $a_{6}(1)$ and further understanding of the singular structure of $a_{6}(\xi)$ requires explicit five- and six-loop computations (at least in a specific gauge).

\section{DIAGRAMS IN THE LANDAU GAUGE}

As we discussed in Sec. II B, there is a total of 87 diagrams to compute in order to derive the fermion propagator of quenched $\mathrm{QED}_{3}$ up to four-loops with an arbitrary gauge-fixing parameter $\xi$. The results presented in Secs. II C and II D, were obtained by computing all of these diagrams.

In order to provide the interested reader with a representative sample of the graphs, we focus in this section on the Landau gauge. The reason is that it is the gauge where there is the least number of diagrams as most of them vanish in the limit $\xi=0$. Moreover, as discussed in Sec. III, it is enough to compute the fermion propagator in this gauge as the LKF transformation allows us to reconstruct the full $\xi$ dependence of the propagator.

In the Landau gauge, there is no one-loop contribution and there are 1, 4, and 30 diagrams at two, three, and four loops, respectively; so there is a total of 35 diagrams. Focusing on the leading order contribution to the $\varepsilon$-expansion of these diagrams, the two- and three-loop contributions will be considered with an accuracy $O(\varepsilon)$ and the four-loop contributions with an accuracy $O\left(\varepsilon^{0}\right)$. So, amongst the 30 four-loop diagrams only the eight divergent ones need to be considered (the other 22 diagrams are finite). Moreover, taking into account the fact that mirror conjugate graphs take the same value, we are left with only three distinct graphs at three loops and four distinct graphs at four loops. Hence a total of eight distinct diagrams contribute to the Landau gauge quenched $\mathrm{QED}_{3}$ fermion propagator up to four loops.

For the sake of clarity, we explicitly display the distinct graphs together with their values. The single diagram contributing at two-loop level is given by

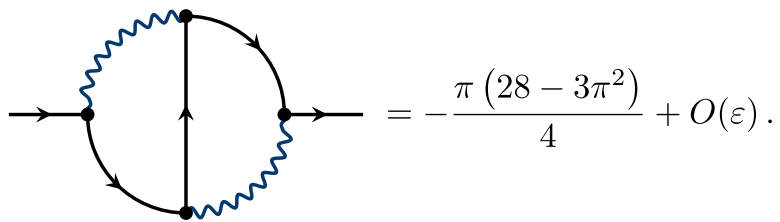

At three loops, the two benz diagrams in (29c) are mirror conjugate to each other and therefore share the same value. Hence, the three distinct three-loop graphs read:
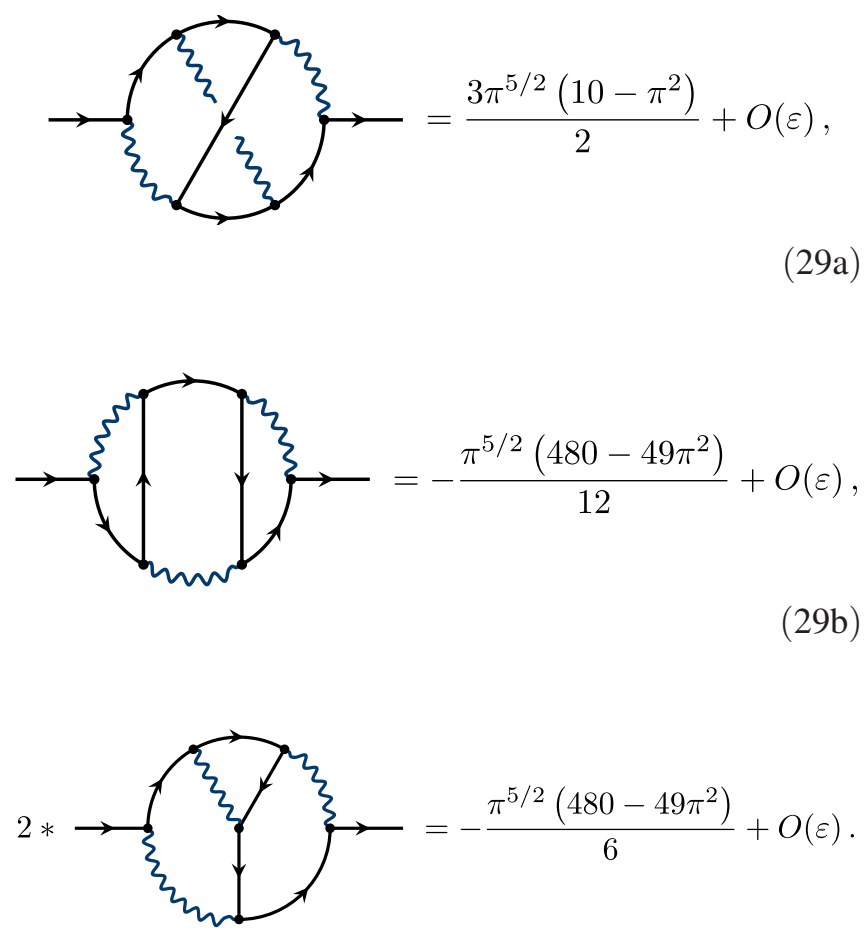

Similarly, the four-loop diagrams are grouped in pairs of mirror conjugate graphs and the four leading contributions are given by
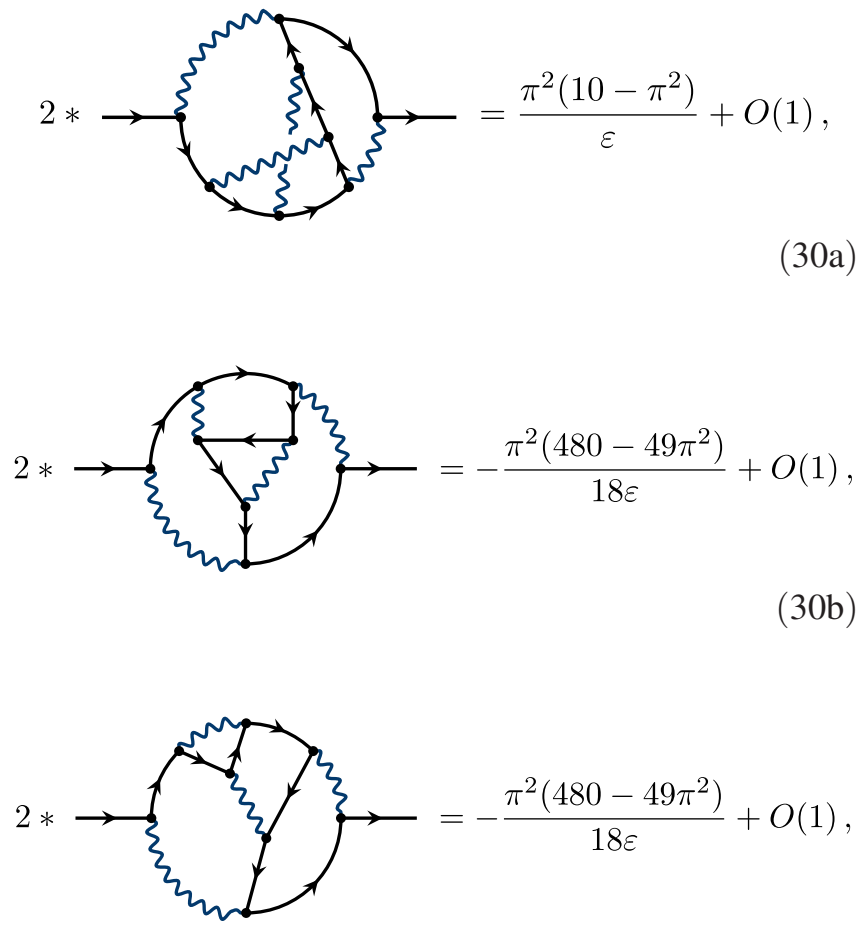


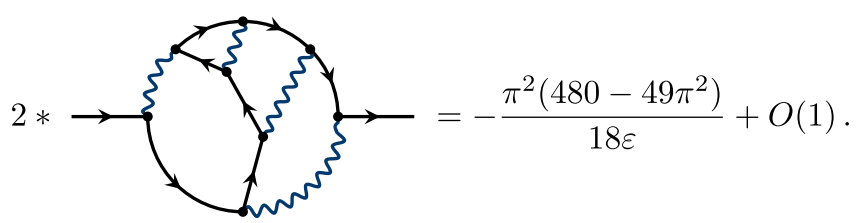

$(30 \mathrm{~d})$

Summing all of the above contributions order by order in the loop expansion yields the coefficients $\sigma_{i}(0)(i=1-4)$ in agreement with Eqs. (10) at the leading order of the $\varepsilon$ expansion. With the accuracy used, these coefficients are equal to the coefficients $a_{i}(0)(i=1-4)$. Substituting them in Eqs. (25) with $\eta=0$ allows us to reconstruct the gaugedependent part of $a_{i}(\xi)(i=1-4)$ in agreement with Eqs. (17) at the leading order of the $\varepsilon$ expansion.

\section{SUMMARY AND CONCLUSION}

In the present paper, we have examined the perturbative structure of the massless fermion propagator of quenched $\mathrm{QED}_{3}$ up to four loops.

Our study was motivated by our recent publication [7] where the gauge covariance of the fermion propagator of quenched $\mathrm{QED}_{3}$ was studied using the LKF transformation in dimensional regularization $(d=3-2 \varepsilon)$. This nonperturbative transformation revealed an interesting parity effect, whereby the contributions of odd orders, starting from the third one, to even orders are accompanied by singularities taking the form of poles, $\varepsilon^{-1}$, in dimensional regularization. In turn, even orders produce contributions to odd ones, starting from the third order, which are $\sim \mathcal{E}$.

Following arguments in favor of the IR (and ultraviolet) perturbative finiteness of massless quenched $\mathrm{QED}_{3}[1,6]$ and therefore assuming the existence of a finite limit as $\varepsilon \rightarrow 0$, we concluded in Ref. [7] that, exactly in $d=3$, all odd coefficients $a_{2 t+1}(\xi)$ in perturbation theory, except $a_{1}$, should be exactly zero in any gauge.

This statement needed a check since analytical expressions for the fermion self-energy diagrams were known only at two-loop order. This is what we have done in the present paper by computing the three- and four-loop corrections to the massless fermion propagator, i.e., the coefficients $a_{3}(\xi)$ and $a_{4}(\xi)$, directly in the framework of perturbation theory (see Sec. IV for some details on the computed diagrams in the Landau gauge). We found that $a_{3}(\xi)$ is finite and gauge-independent when $\varepsilon \rightarrow 0$. The coefficient $a_{4}(\xi)$ is, on the other hand, singular which violates the status of IR perturbative finiteness of massless quenched $\mathrm{QED}_{3}$. The obtained singularity is such that all of its gauge-fixing dependent terms are entirely determined by lower order contributions in agreement with the properties of the LKF transformation.

In closing, let us note that the four-loop singularities were found to contribute to the coefficient $a_{4}(\xi)$ with a factor $\sim(1-\xi)$, and, thus, $a_{4}(\xi)$ is finite in the Feynman gauge. The reason for this intriguing effect is not clear at present and its elucidation requires additional research.

\section{ACKNOWLEDGMENTS}

A. V. K. thanks A. G. Grozin and A. L. Kataev for useful discussions. The work of V.P.G. is supported by the National Academy of Sciences of Ukraine (Project No. 0116U003191) and by its Program of Fundamental Research of the Department of Physics and Astronomy (Project No. 0117U000240). The work of A.F.P. is supported by the Foundation for the Advancement of Theoretical Physics and Mathematics "BASIS."
[1] R. Jackiw and S. Templeton, How superrenormalizable interactions cure their infrared divergences, Phys. Rev. D 23, 2291 (1981).

[2] E. I. Guendelman and Z. M. Radulovic, Loop expansion in massless QED in three-dimensions, Phys. Rev. D 27, 357 (1983); Infrared divergences in three-dimensional gauge theories, Phys. Rev. D 30, 1338 (1984).

[3] S. Templeton, Summation of coupling constant logarithms in QED in three-dimensions, Phys. Rev. D 24, 3134 (1981).

[4] M. C. Bergere and F. David, Nonanalyticity of the perturbative expansion for superrenormalizable massless field theories, Ann. Phys. (N.Y.) 142, 416 (1982).

[5] I. D. King and G. Thompson, Nonperturbative analysis of leading logarithms in three-dimensional QED, Phys. Rev. D 31, 2148 (1985).
[6] N. Karthik and R. Narayanan, Flavor and topological current correlators in parity-invariant three-dimensional QED, Phys. Rev. D 96, 054509 (2017).

[7] V. P. Gusynin, A. V. Kotikov, and S. Teber, LandauKhalatnikov-Fradkin transformation in three-dimensional quenched QED, Phys. Rev. D 102, 025013 (2020).

[8] R. D. Pisarski, Chiral symmetry breaking in threedimensional electrodynamics, Phys. Rev. D 29, 2423 (1984).

[9] T. W. Appelquist, M. J. Bowick, D. Karabali, and L. C. R. Wijewardhana, Spontaneous chiral symmetry breaking in three-dimensional QED, Phys. Rev. D 33, 3704 (1986).

[10] T. Appelquist, D. Nash, and L. C. R. Wijewardhana, Critical Behavior in $(2+1)$-Dimensional QED, Phys. Rev. Lett. 60, 2575 (1988).

[11] D. Nash, Higher Order Corrections in $(2+1)$-Dimensional QED, Phys. Rev. Lett. 62, 3024 (1989). 
[12] D. Atkinson, P. W. Johnson, and P. Maris, Dynamical mass generation in QED in three-dimensions: Improved vertex function, Phys. Rev. D 42, 602 (1990).

[13] M. R. Pennington and D. Walsh, Masses from nothing: A Nonperturbative study of QED in three-dimensions, Phys. Lett. B 253, 246 (1991).

[14] A. V. Kotikov, Critical behavior of 3-D electrodynamics, Pis'ma Zh. Eksp. Teor. Fiz. 58, 785 (1993) [JETP Lett. 58, 731 (1993)]; On the critical behavior of $(2+1)$-dimensional QED, Phys. At. Nucl. 75, 890 (2012).

[15] V. P. Gusynin, A. H. Hams, and M. Reenders, $(2+1)-$ dimensional QED with dynamically massive fermions in the vacuum polarization, Phys. Rev. D 53, 2227 (1996).

[16] P. Maris, The influence of the full vertex and vacuum polarization on the fermion propagator in QED in threedimensions, Phys. Rev. D 54, 4049 (1996).

[17] V. P. Gusynin and M. Reenders, Infrared cutoff dependence of the critical flavor number in QED(3), Phys. Rev. D 68, 025017 (2003).

[18] C. S. Fischer, R. Alkofer, T. Dahm, and P. Maris, Dynamical chiral symmetry breaking in unquenched QED(3), Phys. Rev. D 70, 073007 (2004).

[19] V.P. Gusynin and P. K. Pyatkovskiy, Critical number of fermions in three-dimensional QED, Phys. Rev. D 94, 125009 (2016).

[20] A. V. Kotikov, V. I. Shilin, and S. Teber, Critical behavior of $(2+1)$-dimensional QED: $1 / \mathrm{N}_{f}$ corrections in the Landau gauge, Phys. Rev. D 94, 056009 (2016); Erratum, Phys. Rev. D 99, 119901 (2019).

[21] A. V. Kotikov and S. Teber, Critical behavior of $(2+1)-$ dimensional QED: $1 / N_{f}$ corrections in an arbitrary nonlocal gauge, Phys. Rev. D 94, 114011 (2016); Erratum, Phys. Rev. D 99, 059902 (2019).

[22] N. Karthik and R. Narayanan, Numerical determination of monopole scaling dimension in parity-invariant threedimensional noncompact QED, Phys. Rev. D 100, 054514 (2019).

[23] A. V. Kotikov and S. Teber, Critical behavior of $(2+1)$ dimensional QED: 1/ $N$ expansion, Particles 3, 345 (2020).

[24] N. Dorey and N. E. Mavromatos, QED in three-dimension and two-dimensional superconductivity without parity violation, Nucl. Phys. B386, 614 (1992).

[25] M. Franz and Z. Tesanovic, Algebraic Fermi Liquid from Phase Fluctuations: 'Topological' Fermions, Vortex 'Berryons,' and QE D-3 Theory of Cuprate Superconductors, Phys. Rev. Lett. 87, 257003 (2001).

[26] I. F. Herbut, QED(3) theory of underdoped high temperature superconductors, Phys. Rev. B 66, 094504 (2002).

[27] K. Farakos and N. Mavromatos, Gauge theory approach to planar doped antiferromagnetics and external magnetic fields, Int. J. Mod. Phys. B 12, 809 (1998).

[28] G. W. Semenoff, Condensed Matter Simulation of a Threedimensional Anomaly, Phys. Rev. Lett. 53, 2449 (1984).

[29] V. P. Gusynin, S. G. Sharapov, and J. P. Carbotte, AC conductivity of graphene: From tight-binding model to $2+1$-dimensional quantum electrodynamics, Int. J. Mod. Phys. B 21, 4611 (2007); A. H. Castro Neto, F. Guinea, N. M. R. Peres, K. S. Novoselov, and A. K. Geim, The electronic properties of graphene, Rev. Mod. Phys. 81, 109 (2009); V. N. Kotov, B. Uchoa, V. M. Pereira, F. Guinea, and A.H. Castro Neto, Electron-electron interactions in graphene: Current status and perspectives, Rev. Mod. Phys. 84, 1067 (2012); S. Teber, Field theoretic study of electronelectron interaction effects in Dirac liquids, Habilitation, Paris, LPTHE, arXiv:1810.08428.

[30] E. Marinari, G. Parisi, and C. Rebbi, Computer Estimates of Meson Masses in SU(2) Lattice Gauge Theory, Phys. Rev. Lett. 47, 1795 (1981); H. Hamber and G. Parisi, Numerical Estimates of Hadronic Masses in a Pure SU(3) Gauge Theory, Phys. Rev. Lett. 47, 1792 (1981).

[31] P. I. Fomin, V. P. Gusynin, V. A. Miransky, and Yu. A. Sitenko, Dynamical symmetry breaking and particle mass generation in gauge field theories, Riv. Nuovo Cimento 6, 1 (1983); V. A. Miransky, Dynamical Symmetry Breaking in Quantum Field Theories (World Scientific, Singapore, 1993), p. 533.

[32] C. N. Leung, S. T. Love, and W. A. Bardeen, Spontaneous symmetry breaking in scale invariant quantum electrodynamics, Nucl. Phys. B273, 649 (1986); Aspects of dynamical symmetry breaking in gauge field theories, Nucl. Phys. B323, 493 (1989); B. Holdom, Continuum Limit of Quenched Theories, Phys. Rev. Lett. 62, 997 (1989); U. Mahanta, Critical behavior in quenched QED to all orders in the coupling, Phys. Lett. B 225, 181 (1989).

[33] V. P. Gusynin, A. W. Schreiber, T. Sizer, and A. G. Williams, Chiral symmetry breaking in dimensionally regularized nonperturbative quenched QED, Phys. Rev. D 60, 065007 (1999).

[34] D. Hatton, C. T. H. Davies, B. Galloway, J. Koponen, G. P. Lepage, and A. T. Lytle (HPQCD Collaboration), Charmonium properties from lattice QCD + QED: Hyperfine splitting, $J / \psi$ leptonic width, charm quark mass and $a_{\mu}^{c}$, Phys. Rev. D 102, 054511 (2020).

[35] A. V. Kotikov and S. Teber, Landau-Khalatnikov-Fradkin transformation and the mystery of even $\zeta$-values in Euclidean massless correlators, Phys. Rev. D 100, 105017 (2019); Landau-Khalatnikov-Fradkin transformation and hatted

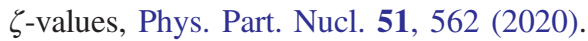

[36] A. James, A. V. Kotikov, and S. Teber, Landau-KhalatnikovFradkin transformation of the fermion propagator in massless reduced QED, Phys. Rev. D 101, 045011 (2020).

[37] L. D. Landau and I. M. Khalatnikov, The gauge transformation of the Green function for charged particles, $\mathrm{Zh}$. Eksp. Teor. Fiz. 29, 89 (1955) [Sov. Phys. JETP 2, 69 (1956)], http://www.jetp.ac.ru/cgi-bin/e/index/e/2/1/p69? $\mathrm{a}=$ list; E. S. Fradkin, Concerning some general relations of quantum electrodynamics, Zh. Eksp. Teor. Fiz. 29, 258 (1955) [Sov. Phys. JETP 2, 361 (1956)], http://www.jetp .ac.ru/cgi-bin/e/index/e/2/2/p361?a=list.

[38] K. Johnson and B. Zumino, Gauge Dependence of the Wave-Function Renormalization Constant in Quantum Electrodynamics, Phys. Rev. Lett. 3, 351 (1959); B. Zumino, Gauge properties of propagators in quantum electrodynamics, J. Math. Phys. (N.Y.) 1, 1 (1960); S. Okubo, The gauge properties of green's functions in quantum electrodynamics, Nuovo Cimento (1955-1965) 15, 949 (1960); I. Bialynicki-Birula, On the gauge properties of green's functions, Nuovo Cimento (1955-1965) 17, 951 (1960); H. Sonoda, On the gauge parameter dependence of QED, Phys. Lett. B 499, 253 (2001). 
[39] A. Bashir, A. Kizilersu, and M. R. Pennington, Does the weak coupling limit of the Burden-Tjiang deconstruction of the massless quenched three-dimensional QED vertex agree with perturbation theory?, Phys. Rev. D 62, 085002 (2000); A. Bashir, Nonperturbative fermion propagator for the massless quenched QED3, Phys. Lett. B 491, 280 (2000); A. Bashir and A. Raya, Landau-Khalatnikov-Fradkin transformations and the fermion propagator in quantum electrodynamics, Phys. Rev. D 66, 105005 (2002).

[40] A. Bashir and A. Raya, Gauge covariance and the chiral condensate in QED3, Braz. J. Phys. 37, 313 (2007).

[41] A. V. Kotikov and S. Teber, Two-loop fermion self-energy in reduced quantum electrodynamics and application to the ultrarelativistic limit of graphene, Phys. Rev. D 89, 065038 (2014).

[42] B. Ruijl, T. Ueda, J. A. M. Vermaseren, and A. Vogt, Fourloop QCD propagators and vertices with one vanishing external momentum, J. High Energy Phys. 06 (2017) 040.

[43] B. Ruijl, T. Ueda, and J. A. M. Vermaseren, Forcer, A FORM program for the parametric reduction of four-loop massless propagator diagrams, Comput. Phys. Commun. 253, 107198 (2020).
[44] R. N. Lee, Space-time dimensionality D as complex variable: Calculating loop integrals using dimensional recurrence relation and analytical properties with respect to $\mathrm{D}$, Nucl. Phys. B830, 474 (2010).

[45] H. Ferguson, D. Bailey, and S. Arno, Analysis of PSLQ, an integer relation finding algorithm, Math. Comput. Am. Math. Soc. 68, 351 (1999).

[46] R. N. Lee, A. V. Smirnov, and V. A. Smirnov, Master integrals for four-loop massless propagators up to transcendentality weight twelve, Nucl. Phys. B856, 95 (2012).

[47] R. N. Lee and K. T. Mingulov, Introducing SummerTime: A package for high-precision computation of sums appearing in DRA method, Comput. Phys. Commun. 203, 255 (2016).

[48] V. Magerya and A. Pikelner, Cutting massless four-loop propagators, J. High Energy Phys. 12 (2019) 026.

[49] A. Kotikov, The Gegenbauer polynomial technique: The evaluation of a class of Feynman diagrams, Phys. Lett. B 375, 240 (1996).

[50] S. Laporta, Building bases for analytical fits of fourloop QED master integrals, Proc. Sci., LL2018 (2018) 073. 